\title{
Diagnostic accuracy of inferior vena cava evaluation in the diagnosis of acute heart failure among dyspneic patients
}

\author{
Alfonso Sforza ${ }^{1}$, Maria Viviana Carlino ${ }^{1}$, Mario Guarino ${ }^{1}$, Ilaria Fucile ${ }^{2}$, Nicola De Luca $^{2}$, \\ Costantino Mancusi ${ }^{2}$ \\ ${ }^{1}$ Emergency Department, CTO Hospital, Naples; ${ }^{2}$ Hypertension Research Center \& Department of Advanced \\ Biomedical Science, Federico II University Hospital, Naples, Italy
}

\begin{abstract}
Acute dyspnea is one of the main reasons for admission to the Emergency Department (ED). A rapid and accurate diagnosis can be lifesaving for these patients. Particularly, it is important to differentiate between dyspnea due to acute heart failure (AHF) and dyspnea of pulmonary origin. The aim of this study is to evaluate the real accuracy of the evaluation of diameter and collapsibility of inferior vena cava (IVC) for the diagnosis of AHF among dyspneic patients. We analyzed 155 patients admitted for acute dysp-
\end{abstract}

Correspondence: Costantino Mancusi, Department of Advanced Biomedical Science, Federico II University Hospital, Via Pansini 5, 80131 Naples, Italy.

Tel. +39.081.7462013 - Fax: +39.081.7462256.

E-mail: costantino.mancusi@unina.it

Key words: Dyspnea; heart failure; IVC; emergency department; point-of-care ultrasound; pocket ultrasound device.

Conflict of interest: The Authors declare no conflict of interest.

Ethics approval and consent to participate: Not applicable, as this study is a post-hoc analysis derived from observational studies; the present study follows the principles of the Declaration of Helsinki.

Informed consent: obtained from each patient included in the study.

Patient consent for publication: Not applicable.

Availability of data and material: The data used to support the findings of this study are available from the corresponding author upon request.

Authors' Contributions: AS, MVC, CM, performed the analysis and draft the manuscript; MG, IF, NDL, critically revised the paper. All the authors have read and approved the final version of the manuscript and agreed to be accountable for all aspects of the work.

Received for publication: 12 May 2020.

Accepted for publication: 31 August 2020.

${ }^{\text {C }}$ Copyright: the Author(s), 2020

Licensee PAGEPress, Italy

Monaldi Archives for Chest Disease 2020; 90:1375

doi: 10.4081/monaldi.2020.1375

This article is distributed under the terms of the Creative Commons Attribution Noncommercial License (by-nc 4.0) which permits any noncommercial use, distribution, and reproduction in any medium, provided the original author(s) and source are credited. nea to the ED of "Maurizio Bufalini" hospital in Cesena (Italy) and "Antonio Cardarelli" hospital in Naples (Italy) from November 2014 to April 2017. All patients underwent ultrasound of IVC examination with a hand-held device in addition to the traditional pathway. Patients were classified into AHF group or nonAHF group according to the current guidelines. The final diagnosis was AHF in 64 patients and dyspnea of non-cardiac origin in 91 patients. Sensibility and specificity of IVC hypo-collapsibility was $75.81 \%(95 \%$ CI $63.26 \%$ to $85.78 \%)$ and $67.74 \%(95 \%$ CI $57.25 \%$ to $77.07 \%$ ) for the diagnosis of AHF. Sensibility and specificity of IVC dilatation was $69.35 \%$ (95\% CI $56.35 \%$ to $80.44 \%$ ) and $74.19 \%$ (95\%CI $64.08 \%$ to $82.71 \%$ ) for the diagnosis of AHF. AUC was 0.718 (0.635-0.801) for IVC hypo-collapsibility, 0.718 (0.634-0.802) for IVC dilatation. Our study demonstrated that the sonographic assessment of IVC diameter and collapsibility is suboptimal to differentiate acute dyspnea due to AHF or other causes in the emergency setting.

\section{Introduction}

Acute heart failure (AHF) is one of the most common reasons for admission to the Emergency Department (ED) and dyspnea is a frequent clinical presentation [1]. A rapid diagnostic pathway is mandatory to establish the correct sequence of therapeutic actions. History, physical examination, ECG, chest X-ray, laboratory tests and multi-organ ultrasound compose the diagnostic workup using which the emergency physician interprets the clinical picture and makes therapeutic decisions. In the last years multi-organ ultrasound examination of the lung, heart and inferior vena cava (IVC) has become a useful tool for the differential diagnosis of acute dyspnea in the ED [2-4]. Since echocardiographic evaluation requires expertise and training, some studies suggest that the only sonographic assessment of IVC diameter and/or collapsibility, from which central venous pressure (CVP) can be estimated, may be enough for the identification of AHF among acutely dyspneic patients [5]. The aim of this study is to evaluate the real accuracy of the evaluation of diameter and collapsibility of IVC for the diagnosis of AHF and to evaluate the sensibility and specificity of different values of CVP for the diagnosis of AHF.

\section{Methods}

This study is a post-hoc analysis derived from two observational studies conducted in the ED of "Maurizio Bufalini" hospital 
in Cesena (Italy) and "Antonio Cardarelli" hospital in Naples (Italy) from November 2014 to April 2017 [6,7]. One hundred and seventy (170) patients admitted to the ED for acute dyspnea or worsening of chronic dyspnea within the previous 48 hours (excluding dyspnea of traumatic origin) underwent multi-organ ultrasound of lung, heart and IVC performed with a handheld ultrasound device in addition to the traditional diagnostic pathway (clinical exam, blood gas analysis, chest X-ray, ECG, routine blood tests) [8]. NT-pro-BNP was considered negative if less than $300 \mathrm{pg} / \mathrm{ml}$ and in favor of AHF if greater than $1800 \mathrm{pg} / \mathrm{ml}$ in patients older than 75 years, $900 \mathrm{pg} / \mathrm{ml}$ in patients from 50 to 75 years old, $450 \mathrm{pg} / \mathrm{ml}$ in patients younger than 50 years.

Informed consent was obtained from each patient included in the study, which follows the principles of the Declaration of Helsinki.

IVC evaluation was performed with a pocket ultrasound device (Vscan of General Electric Healthcare) with a single probe (1.7-3.8 $\mathrm{MHz}$ ), using cardiac preset in sub-costal window [9]. Off-line measures for IVC diameter and collapsibility were performed. The presence of IVC dilatation was established if IVC diameter was $>2 \mathrm{~cm}$ while the presence of hypo-collapsibility was established if the variation between expiratory and inspiratory diameter of IVC was $\leq 50 \%$ (Video 1). Four levels of estimated right atrial pressure (eRAP) were identified according with the combination of IVC maximum diameter and collapsibility based on current guidelines [10]. Ejection fraction $(\mathrm{EF})$ was estimated visually and categorized as preserved if $>40 \%(\mathrm{n}=100)$ or reduced if $\leq 40 \%(\mathrm{n}=55)$ from apical view [9].

The final diagnosis, considered as the gold standard, was issued by two independent observers (one emergency physician and one internal medicine specialist) who had access to the entire medical chart (from emergency department admission to hospital discharge) of each patient. Patients were classified into AHF group or non-AHF group according to the current guidelines [11]. In patients with coexistence of heart failure and another cause of dyspnea the main diagnosis was considered AHF. In the present analysis 155 patients with available ultrasound imaging of IVC have been included. Data were analyzed using SPSS version 21.0 (SPSS, Chicago, Illinois, USA). Continuous data are expressed as mean $\pm 1 \mathrm{SD}$ and categorical variables as percentages. Quantitative variables were compared by using Student's $t$-test while $\chi 2$ distribution was used to compare categorical variables. Multivariate logistic regression model was built to assess the association of age, sex, NT-proBNP level, creatinine and IVC collapsibility for the diagnosis of AHF. A p-level $<0.05$ was considered statistically significant.

\section{Results}

The final diagnosis was AHF in 64 patients and dyspnea of non-cardiac origin in 91 patients. Baseline characteristics of the study population based on diagnosis of AHF is reported in Table 1. Sensibility and specificity of IVC hypo-collapsibility was $75.81 \%$ (95\% CI $63.26 \%$ to $85.78 \%$ ) and $67.74 \%$ (95\% CI $57.25 \%$ to $77.07 \%$ ) for the diagnosis of AHF. Sensibility and specificity of IVC dilatation was $69.35 \%$ (95\% CI $56.35 \%$ to $80.44 \%$ ) and $74.19 \%$ (95\%CI $64.08 \%$ to $82.71 \%$ ) for the diagnosis of AHF. Sensibility and specificity for different levels of eRAP are reported in Table 2. AUC was 0.718 (0.635-0.801) for IVC hypo-collapsibility, 0.718 (0.634-0.802) for IVC dilatation, 0.737(0.653-0.820) for $\mathrm{eRAP} \geq 15 \mathrm{mmHg}$. In multivariate logistic regression model NT-proBNP level adjusted for age and IVC collapsibility are strongly and independently associated with AHF (Table 3). The study population was than divided based on preserved and reduced EF. Sensibility and specificity for IVC hypo-collapsibility and dilatation are reported in Table 4.AUC for preserved or reduced EF was respectively $0.675(0.536-0.814)$ for IVC hypo-collapsibility and $0.717(0.580-0.855)$ for IVC dilatation, $0.748(0.567-0.928)$ for IVC hypo-collapsibility and $0.652(0.458-0.847)$ for IVC dilatation.

\section{Discussion}

In patients with HF the presence of an elevated jugular venous pulse is one of the key semiotic findings and reflects the presence of elevated CVP. More accurately CVP can be estimated using ultrasound examination of IVC [12]. Patients with AHF are characterized by larger IVC diameter and reduced collapsibility compared to non AHF patients, even though increased CVP may not be always present [5]. Different studies have evaluated the diagnostic accuracy of IVC for the diagnosis of AHF among patients with acute dyspnea. In 2014 Yamanoglu et al. conducted a prospective observational study to distinguish between dyspnea of cardiac origin and dyspnea of pulmonary origin using the IVC diameter measurement. They found that IVC diameter above $18.3 \mathrm{~mm}$ have $81.3 \%$ sensibility and $73.8 \%$ specificity for the diagnosis of AHF [13]. These findings are in line with our results and the slightly better diagnostic accuracy should be explained by the better accuracy of their echocardiographic measurement and by the intensive care setting of the study.

Combined information from IVC diameter and collapsibility using a sonographic caval index (calculated as the percentage decrease in the IVC diameter during respiration) was demonstrated to be a useful clinical adjunct to establish the diagnosis of AHF in patients with undifferentiated dyspnea [14]. In particular low caval index has high specificity for the diagnosis of $\mathrm{HF}$, in line with our analysis related to eRAP $>15 \mathrm{mmHg}$. NT-ProBNP and IVC collapsibility are strongly and independently associated with AHF, demonstrating that fluid overload and ventricular stretching are the main pathophysiological determinant of AHF [15].

In patients with chronic heart failure (CHF) IVC diameter and collapsibility have strong correlation with volume status, natriuretic peptide and give important prognostic information $[15,16]$. Thus when the cause of acute dyspnea is decompensation of CHF IVC diameter can be useful for diagnostic and therapeutic approach $[17,18]$. Differently some patients, presenting with dyspnea due to AHF, do not have volume overload but can be affected by acute pressure overload such us in patients with hypertensive emergency. In hypertensive AHF CVP can be normal while there is a redistribution of existing fluids from the splanchnic and central circulations into the pulmonary vasculature [19]. The main cause of dyspnea in AHF is the increase in pulmonary wedge pressure due to increased afterload and volume-shift lung congestion which is not always associated with increased right atrial pressure. This is particularly true among patients with new onset AHF compared to patient with chronic HF [20]. This explains way echocardiographic assessment of IVC cannot be alone the diagnostic method of choice for the diagnosis of AHF in patients with acute dyspnea.

Our study demonstrated that the diagnostic accuracy of IVC evaluation in predicting AHF is similar among patients with preserved or reduced EF. This is in line with recent evidence form Van Aelst et al. who demonstrated similar echocardiographic and biomarkers signs of venous congestions in patients with heart failure with preserved or reduced EF [21]. 
Another aspect to bear in mind is that elevated right atrial pressure can also be a marker of acute respiratory disease such as pulmonary embolism or pneumothorax or chronic lung diseases in which dilated and not collapsing IVC do not reflect AHF but just increased pulmonary artery pressure or intrathoracic pressure.

In conclusion our study demonstrated that the sonographic evaluation of IVC diameter or collapsibility alone for the diagnosis of AHF among dyspneic patients is suboptimal. Combining infor-

Table 1. Baseline characteristics and clinical findings detected at the time of patient presentation in the emergency department.

\begin{tabular}{|c|c|c|c|}
\hline & Acute heart failure $(n=64)$ & Non acute heart failure $(n=91)$ & p value \\
\hline Age (years) & $75.8 \pm 11.1$ & $73.5 \pm 13.3$ & 0.21 \\
\hline Women (\%) & 44 & 41 & 0.68 \\
\hline Medical history of chronic obstructive pulmonary disease (\%) & 23 & 49 & 0.001 \\
\hline Medical history of heart failure and/or ischemic heart disease (\%) & 41 & 18 & 0.01 \\
\hline Heart rate (bpm) & $90.5 \pm 25.5$ & $94.2 \pm 21.4$ & 0.62 \\
\hline Systolic BP (mmHg) & $141.2 \pm 30.7$ & $132.8 \pm 22.2$ & 0.01 \\
\hline Diastolic BP (mmHg) & $79.7 \pm 15.6$ & $73.7 \pm 12.7$ & 0.09 \\
\hline Serum creatinine (mg/dl) & $1.6 \pm 1.7$ & $1.1 \pm 0.5$ & 0.0001 \\
\hline White blood cell count $\left(\mathrm{x} 10^{3} / \mu \mathrm{l}\right)$ & $9.5 \pm 4.5$ & $11.9 \pm 6.1$ & 0.002 \\
\hline Oxygen saturation (\%) & $91.8 \pm 5.0$ & $89.8 \pm 9.0$ & 0.005 \\
\hline $\mathrm{PaO}_{2} / \mathrm{FiO}_{2}$ & $276.2 \pm 88.7$ & $250.4 \pm 85.5$ & 0.84 \\
\hline $\mathrm{pH}$ & $7.42 \pm 0.09$ & $7.41 \pm 0.09$ & 0.67 \\
\hline Lactate level $(\mathrm{mmol} / \mathrm{l})$ & $1.94 \pm 2.19$ & $1.68 \pm 1.13$ & 0.09 \\
\hline NT-pro BNP (pg/dl) & $9350 \pm 11450$ & $2984 \pm 7034$ & 0.007 \\
\hline Ejection fraction (\%) & $42 \pm 14$ & $57 \pm 8$ & 0.0001 \\
\hline Dilated left atrium (\%) & 87 & 25 & 0.0001 \\
\hline
\end{tabular}

Table 2. Sensibility and specificity for different values of eRAP for the diagnosis of acute heart failure.

\begin{tabular}{lll} 
& Sensibility (\%) & Specificity (\%) \\
eRAp $\geq 10 \mathrm{mmHg}$ & $80.65(68.63$ to 89.58$)$ & $59.14(48.46$ to 69.23$)$ \\
eRAp $\geq 15 \mathrm{mmHg}$ & $64.52(51.34$ to 76.26$)$ & $82.80(73.5$ to 89.83$)$ \\
\hline
\end{tabular}

Table 3. Multivariable regression model for main determinant of acute heart failure.

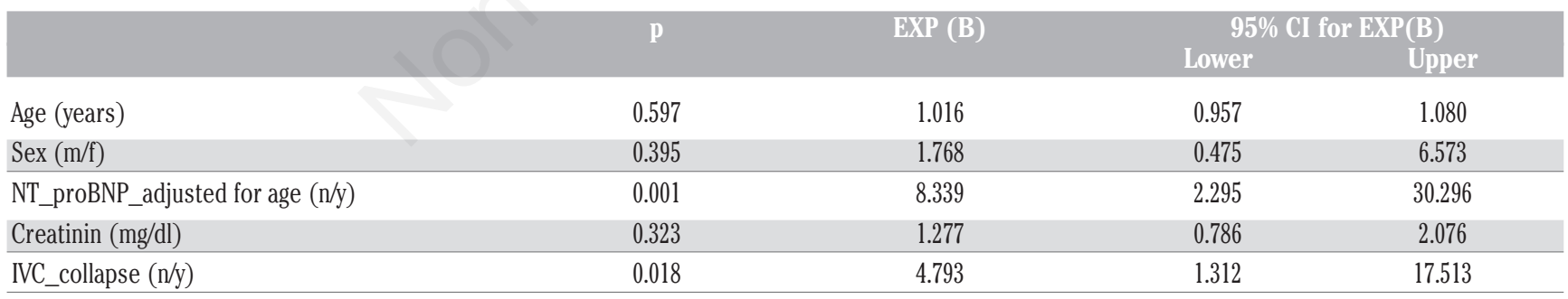

Table 4. Sensibility and specificity of IVC hypo-collapsibility and dilatation in patients with preserved and reduced ejection fraction.

\begin{tabular}{lll}
\hline & Sensibility (\%) & Specificity (\%) \\
\hline Preserved EF & & $68.29(57.08$ to 78.13$)$ \\
IVC hypo-collapsibility & $66.67(40.99$ to 86.66$)$ & $76.83(66.20$ to 85.44$)$ \\
IVC dilatation & $66.67(40.99$ to 86.66$)$ & $70.00(34.75$ to 93.33$)$ \\
\hline Reduced EF & & $60.00(26.24$ to 87.84$)$ \\
\hline IVC hypo-collapsibility & $79.55(64.70$ to 90.20$)$ & $70.45(54.80$ to 83.24$)$ \\
\hline IVC dilatation &
\end{tabular}

EF, ejection fraction; IVC, inferior vena cava. 
mation of IVC collapsibility and diameter helps to improve the diagnostic accuracy which remains suboptimal and does not differ significantly among patients with reduced of preserved EF.

\section{References}

1. Kurmani S, Squire I. Acute heart failure: Definition, classification and epidemiology. Curr Heart Fail Rep 2017:385-92.

2. Pivetta E, Goffi A, Lupia E, et al. Lung Ultrasound-implemented diagnosis of acute decompensated heart failure in the ED: A SIMEU multicenter study. Chest 2015:202-10.

3. Anderson KL, Jenq KY, Fields JM, et al. Diagnosing heart failure among acutely dyspneic patients with cardiac, inferior vena cava, and lung ultrasonography. Am J Emerg Med 2013:1208-14.

4. Sforza A, Carlino MV, Guarino M, et al. A case of pulmonary edema: The critical role of lung-heart integrated ultrasound examination. Monaldi Arch Chest Dis 2018;88:982. doi: 10.4081/monaldi.2018.982

5. Darwish OS, Mahayni A, Kataria S, et al. Diagnosis of acute heart failure using inferior vena cava ultrasound: Systematic review and meta-analysis. J Ultrasound Med 2020;39:1367-78.

6. Sforza A, Mancusi C, Carlino MV, et al. Diagnostic performance of multi-organ ultrasound with pocket-sized device in the management of acute dyspnea. Cardiovasc Ultrasound 2017;15:16.

7. Carlino MV, Paladino F, Sforza A, et al. Assessment of left atrial size in addition to focused cardiopulmonary ultrasound improves diagnostic accuracy of acute heart failure in the Emergency Department. Echocardiography 2018;35:785-91.

8. Sforza A, Carlino MV, Guarino M, et al.. Anterior vs lateral symmetric interstitial syndrome in the diagnosis of acute heart failure. Int J Cardiol 2019;280:130-2.

9. Mancusi C, Carlino MV, Sforza A. Point-of-care ultrasound with pocket-size devices in emergency department. Echocardiography 2019;36:1755-64.

10. Lang RM, Badano LP, Mor-Avi V, et al. Recommendations for cardiac chamber quantification by echocardiography in adults: an update from the American Society of Echocardiography and the European Association of Cardiovascular Imaging. Eur Heart J Cardiovasc Imaging 2015;28:233-70.

11. Ponikowski P, Voors AA, Anker SD, et al. 2016 ESC
Guidelines for the diagnosis and treatment of acute and chronic heart failure: The Task Force for the diagnosis and treatment of acute and chronic heart failure of the European Society of Cardiology (ESC) Developed with the special contribution of the Heart Failure Association (HFA) of the ESC. Eur Heart J 2016;37:2129-200.

12. Brennan JM, Blair JE, Goonewardena S, et al. A comparison by medicine residents of physical examination versus handcarried ultrasound for estimation of right atrial pressure. Am J Cardiol 2007;99:1614-6.

13. Yamanoğlu A, Çelebi Yamanoğlu NG, Parlak İ, et al. The role of inferior vena cava diameter in the differential diagnosis of dyspneic patients; best sonographic measurement method? Am J Emerg Med 2015;33:396-401.

14. Miller JB, Sen A, Strote SR, et al. Inferior vena cava assessment in the bedside diagnosis of acute heart failure. Am J Emerg Med 2012;30:778-83.

15. Curbelo J, Aguilera M, Rodriguez-Cortes $P$, et al. Usefulness of inferior vena cava ultrasonography in outpatients with chronic heart failure. Clin Cardiol 2018;41:510-7.

16. Akhabue E, Pierce JB, Davidson LJ, et al. A prospective pilot study of pocket-carried ultrasound pre- and post-discharge inferior vena cava assessment for prediction of heart failure rehospitalization. J Card Fail 2018;24:614-7.

17. Besli F, Kecebas M, Caliskan S, et al. The utility of inferior vena cava diameter and the degree of inspiratory collapse in patients with systolic heart failure. Am J Emerg Med 2015;33:653-7.

18. Tchernodrinski S, Lucas BP, Athavale A, et al. Inferior vena cava diameter change after intravenous furosemide in patients diagnosed with acute decompensated heart failure. J Clin Ultrasound 2015;43:187-93.

19. Asahi T, Nakata M, Higa N, et al. Respiratory collapse of the inferior vena cava reflects volume shift and subsequent fluid refill in acute heart failure syndrome. Circ J 2016;80:1171-7.

20. Ferreira J. Vascular phenotypes of acute decompensated vs. new-onset heart failure: treatment implications. ESC Heart Fail 2017;4:679-85.

21. Van Aelst LNL, Arrigo M, Placido R, et al. Acutely decompensated heart failure with preserved and reduced ejection fraction present with comparable haemodynamic congestion. Acutely decompensated heart failure with preserved and reduced ejection fraction present with comparable haemodynamic Eur J Heart Fail 2018;20:738-47. 